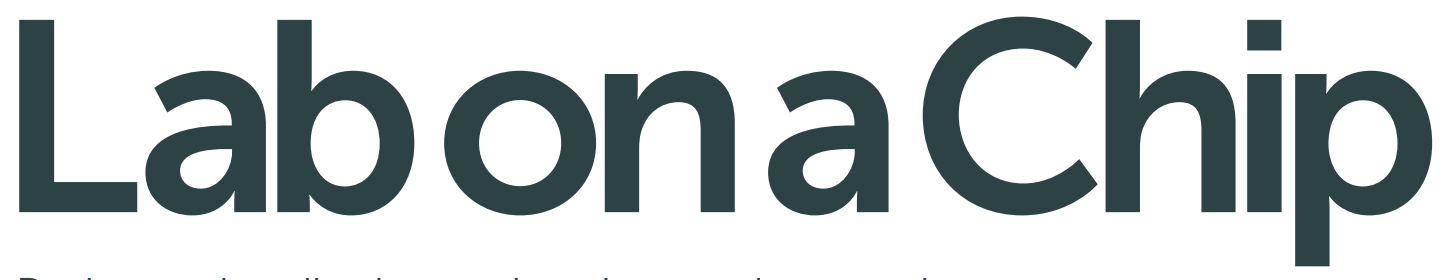

Devices and applications at the micro- and nanoscale rsc.li/loc

\title{
ISSN 1473-0197
}


Check for updates

Cite this: Lab Chip, 2019, 19, 1352

\title{
High-throughput single-particle detections using a dual-height-channel-integrated pore $\dagger$
}

\author{
Wataru Tonomura, ${ }^{\text {a }}$ Makusu Tsutsui, (DD *a Akihide Arima, (D) Kazumichi Yokota, ${ }^{\text {ab }}$ \\ Masateru Taniguchi, ${ }^{a}$ Takashi Washio ${ }^{a}$ and Tomoji Kawai ${ }^{\star a}$
}

\begin{abstract}
We report a proof-of-principle demonstration of particle concentration to achieve high-throughput resistive pulse detections of bacteria using a microfluidic-channel-integrated micropore. We fabricated polymeric nanochannels to trap micrometer-sized bioparticles via a simple water pumping mechanism that allowed aggregation-free size-selective particle concentration with negligible loss. Single-bioparticle detections by ionic current measurements were then implemented through releasing and transporting the thus-collected analytes to the micropore. As a result, we attained two orders of magnitude enhancement in the detection throughput by virtue of an accumulation effect via hydrodynamic control. The device concept presented may be useful in developing nanopores and nanochannels for high-throughput single-particle and -molecule analyses.
\end{abstract}

Received 16th December 2018 Accepted 16th February 2019

DOI: 10.1039/c8lc01371c

rsc.li/loc
In this context, extensive research efforts have been devoted to incorporating additional structures and probes to improve the particle detection efficiency of solid-state pore sensors. Multipore structures were reported to render increased detection throughput. ${ }^{12-14}$ The electric field, ${ }^{15,16}$ laser light, ${ }^{17}$ temperature gradient, ${ }^{18,19}$ and salt-gradient ${ }^{20}$ were also found to serve as effective driving forces to expedite the capture of analytes into nanopores. However, these concepts generally involve side effects on the ionic current blockade through cross-pore interactions and electric field cross-talk. ${ }^{14}$ In contrast, hydrodynamic control of water flow is a more direct and simple way of regulating mass transport outside the pores with little interference with the resistive pulse measurements. ${ }^{21}$ Here we report a nanochannel-integrated pore sensor for high-throughput single-particle detections. It uses a fluidic channel array to filter oversized particles in liquid via fluid flow manipulations that also enabled size-dependent and aggregation-free concentration with a negligible loss of analytes. By this, we demonstrate two orders of magnitude enhancement in the detection throughput for Escherichia coli in an electrolyte solution.

\section{Experimental}

Fabrication of dual-height fluidic channels

$500 \mathrm{~nm}$ thick photo-resist SU-8 was spin-coated on a coated Si wafer. Arrays of $5 \mu \mathrm{m}$-wide lines were patterned with a 15 $\mu \mathrm{m}$ pitch by a light-emitting diode (LED) lithography process. Subsequently, $30 \mu \mathrm{m}$ thick SU-8 was over-coated and a main channel and rest of the structure were delineated by LED lithography. After development, we obtained an SU-8 mold of dual-height fluidic channel structure. On the mold substrate, 
we cured polydimethylsiloxane (PDMS; Sylgard184, Dow Corning) which was baked at 100 degrees Celsius for 24 hours. By cutting into a $20 \mathrm{~mm} \times 20 \mathrm{~mm}$ size and peeling-off, we obtained a PDMS block with dual-height channels formed on one side.

\section{Formation of $\mathrm{Si}_{3} \mathrm{~N}_{4}$ micropores}

A $300 \mu \mathrm{m}$-thick Si wafer, both sides coated with low-pressure chemical vapour deposition-grown 50 nm-thick $\mathrm{Si}_{3} \mathrm{~N}_{4}$ layers, was diced into $25 \mathrm{~mm} \times 25 \mathrm{~mm}$ pieces. On the Si chip, we placed a metal mask and etched-out the exposed $\mathrm{Si}_{3} \mathrm{~N}_{4}$ of 1 $\mathrm{mm} \times 1 \mathrm{~mm}$ area on one side. Then, the chip was immersed in a $25 \% \mathrm{KOH}$ solution (Aldrich) heated at 80 degrees Celsius for deep etching of Si. By completely dissolving the Si layer, we formed a $100 \mu \mathrm{m} \times 100 \mu \mathrm{m}$ sized $\mathrm{Si}_{3} \mathrm{~N}_{4}$ membrane of approximately $40 \mathrm{~nm}$ thickness. On the membrane, we spin coated an electron beam resist (ZEP520A-7, Zeon) and drew a micropore by electron-beam lithography. After development, we opened the pore by reactive ion etching of $\mathrm{Si}_{3} \mathrm{~N}_{4}$ using $\mathrm{CHF}_{3}$ as the etchant gas. Finally, the residual resist was removed by keeping the chip in $N, N$-dimethylformamide (Wako) overnight followed by rinsing in isopropanol, ethanol, and acetone.

\section{Integration of fluidic channels and a micropore}

To the micropore chip, we adhered a piece of Kapton tape to protect a portion of the $\mathrm{Si}_{3} \mathrm{~N}_{4}$ layer during the under-mentioned plasma treatment so as to keep the local region relatively hydrophobic to block water flow into the pore during the particle concentration through hydrodynamic control. Both the micropore chip and the PDMS block were exposed to oxygen plasma for surface activation. The surfaces were then put together for eternal bonding under an optical microscope to align both structures. In the PDMS block, several holes were punched that were used as solution inlets and outlets as well as a place to insert $\mathrm{Ag} / \mathrm{AgCl}$ rods for the ionic current measurements.

\section{Hydrodynamic control}

Teflon tubes were inserted into the inlet and outlet holes in the PDMS block, whose other ends were connected to a pressure control system (OB1, Elveflow). This device was arranged to quicken the draining of the particle-containing PBS buffer capillary-injected into the side channel array via the main channel at a negative hydro-pressure of $-70 \mathrm{kPa}$ during the particle concentration stage. After allowing the entire liquid to flow, we released the pressure to repel the trapped particles from the side channel openings. The particles were then transported to the micropore for resistive pulse detections.

\section{Ionic current measurements}

Two Ag/AgCl electrodes were placed at the top and bottom of the micropore. A bias voltage of $0.1 \mathrm{~V}$ was applied to the membrane and the ionic current through the pore was recorded at a $1 \mathrm{MHz}$ sampling rate by using a custom-built current amplifier and a fast digitizer (NI-5922, National Instruments).

\section{Finite element analysis}

Pressure-driven water flow along with particle motions in the fluidic channels was simulated using COMSOL. ${ }^{17}$ We defined three-dimensional models of the $500 \mathrm{~nm}$-high side channels of lengths $100 \mu \mathrm{m}, 50 \mu \mathrm{m}$, and $30 \mu \mathrm{m}$ connected to reservoirs. The pressure was set as $-70 \mathrm{kPa}$ and $0 \mathrm{kPa}$ at the upper and the lower ends. The induced fluid speed was obtained through solving the Navier-Stokes equation under an assumption of lamellar flow. Bulk physical properties were used for material parameters.

\section{Results and discussion}

\section{Structure of a fluidic-channel-integrated micropore}

Our device concept is based on trapping oversized particles at shallow channels (Fig. 1a and b). Specifically, we employed $500 \mathrm{~nm}$-thick and $5 \mu \mathrm{m}$-wide channel arrays, which we call side channels (Fig. 1c and d). Pressure-assisted capillary flow was induced through these conduits (Fig. 2a). When the solution contains objects larger than the height of the channels, they tend to be trapped at the entrance while smaller particles flow through with the fluid (Fig. 2b, see also Fig. 1a right inset). In this way, we can not only concentrate analytes but at the same time filter small contaminants by a simple hydrodynamic control. ${ }^{22}$ Here, an important design parameter is the length of the main and side channels. The former cannot be made too short so as to ensure that all the particles will be captured at the side channels, while the latter should be made short to mitigate the pressure loss for shortening the time to complete the concentration process. For this, we investigated the influence of the side channel length $L_{\mathrm{s}}$ on the particle trap efficiency while fixing the channel height to $500 \mathrm{~nm}$ (Fig. 1d). At the main channel downstream, there was a micropore of diameter $d_{\text {pore }}$ formed in a $50 \mathrm{~nm}$-thick $\mathrm{Si}_{3} \mathrm{~N}_{4}$ membrane (Fig. 1a, left inset). After releasing the trapped particles (Fig. 2c), they were transported via water flow control toward this micropore for single-particle detections by cross-pore ionic current measurements (Fig. 2d).

\section{Particle concentration via hydrodynamic control in a microchannel system}

Using the nanochannel-micropore structure, we examined particle trapping-to-release via hydro-pressure control. For this, we prepared a dilute suspension of $6 \mu \mathrm{m}$-sized carboxylated polystyrene beads in $0.1 \times$ PBS at a concentration of $10^{4}$ particles per $\mathrm{mL}$. When a negative pressure $P$ of $-70 \mathrm{kPa}$ was applied across the side channel, we observed fast-moving microbeads under an optical microscope that tend to approach and become immobilized at the entrance of one of the side channels (Fig. 3a, see also Movie S1†). The channel to be trapped seemed to be random, reflecting the stochastic nature of the particle dynamics. Meanwhile, there was an 
a

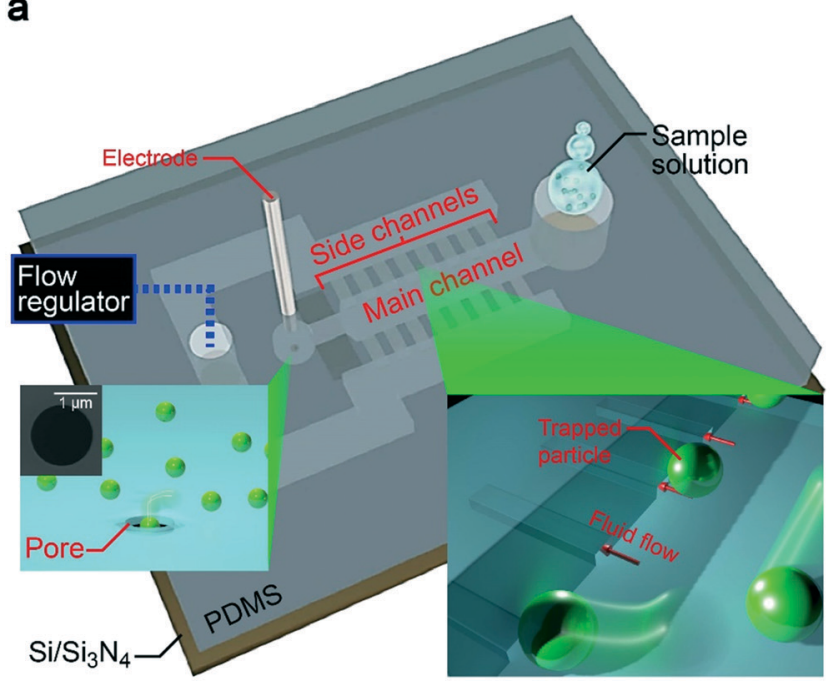

b

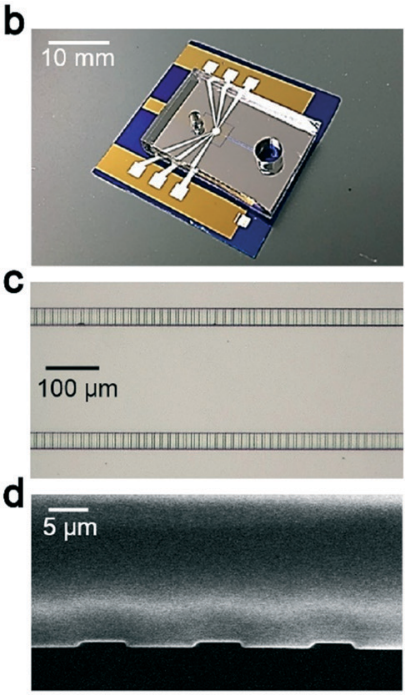

Fig. 1 Dual-height-channel-integrated pore sensor. a, Schematic model of the sensor structure composed of a PDMS block with dual-height fluidic channels adhered to a $\mathrm{Si} / \mathrm{Si}_{3} \mathrm{~N}_{4}$ substrate with a micropore (inset: scanning electron microscopy image) for single-particle detections via crossmembrane ionic current measurements using $\mathrm{Ag} / \mathrm{AgCl}$ electrodes. The fluidic channels consist of a main channel of microscale height and 500 $\mathrm{nm}$-high side channels. The chip is connected to a flow regulator for pumping the injected solution. b, Photo-image of the channel-integrated pore sensor. c and d, Optical microscopy image (c) and cross-sectional SEM observation (d) of the side channels.

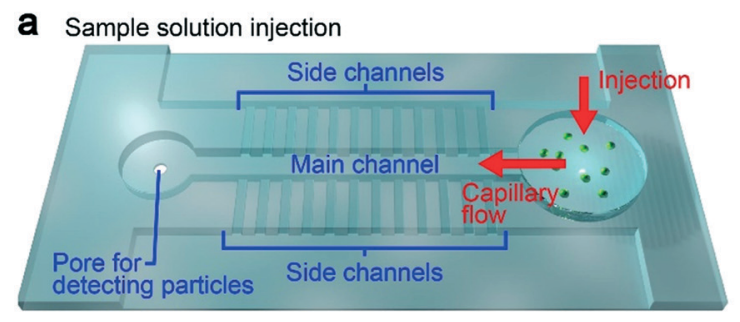

a Sample solution injection

C Particle release

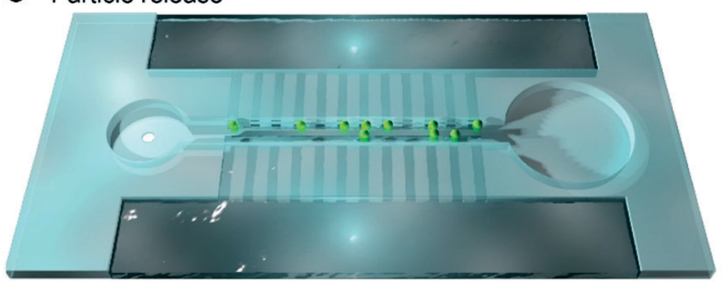

b Particle filter/trap

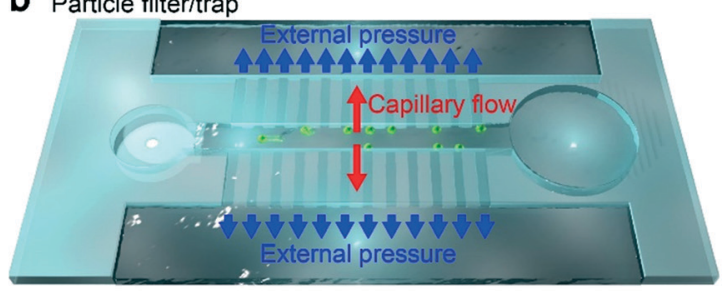

d

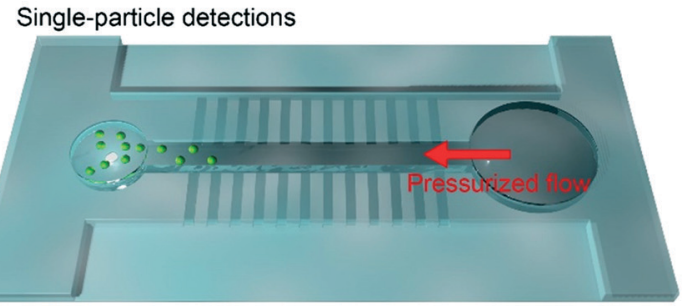

Fig. 2 Particle concentration via fluid dynamics control in a dual-height channel system. a, A dilute suspension of particles is capillary-injected into the main channel. b, After adding an external pressure, the solution is flowed through the side channels. Particles larger than the height of the side channels are trapped at the orifice. A hydrophobic layer at the back-end of the main channel (not shown) prevents the solution from being poured into the pore chamber. c, After draining the entire amount of the solution, the pressure is released to release the trapped particles into the main channel. $d$, The particles are then pushed to the chamber with a pore for single-particle detections. Here, by minimizing the volume of the solution, a high concentration rate can be achieved.

overall trend that particles initially moving close to (away from) the main channel center tend to be captured at a side channel at the back (front). Owing to this feature, the particles less frequently get in contact with each other indicating negligible aggregation.

After draining the entire amount of solution, the particles were all trapped at the side channels with none of them left in the main channel which indicates $100 \%$ capture efficiency when ignoring the possibility of non-specific adhesion in non-observed regions such as the inlet hole and the Teflon tubes (Fig. 3b; see also Table $\mathrm{S} 1 \dagger$ ). To release the polymeric beads for post analysis, we subsequently turned-off the pressure and pushed the beads into the main channel (Fig. 3c). This was, however, found to be critically difficult in asfabricated microchannels wherein we observed more than $86 \%$ of the particles being non-released due presumably to non-specific binding to the hydrophobic PDMS surface (ESI $\dagger$ Fig. S1). ${ }^{23}$ In stark contrast, the polystyrene beads became easily releasable when the channel surface was pre-treated with oxygen plasma (Fig. 3c; see also Movie S2†). It can be interpreted as a consequence of the created silanol group ${ }^{24,25}$ that not only offered hydrophilicity but also negative surface 

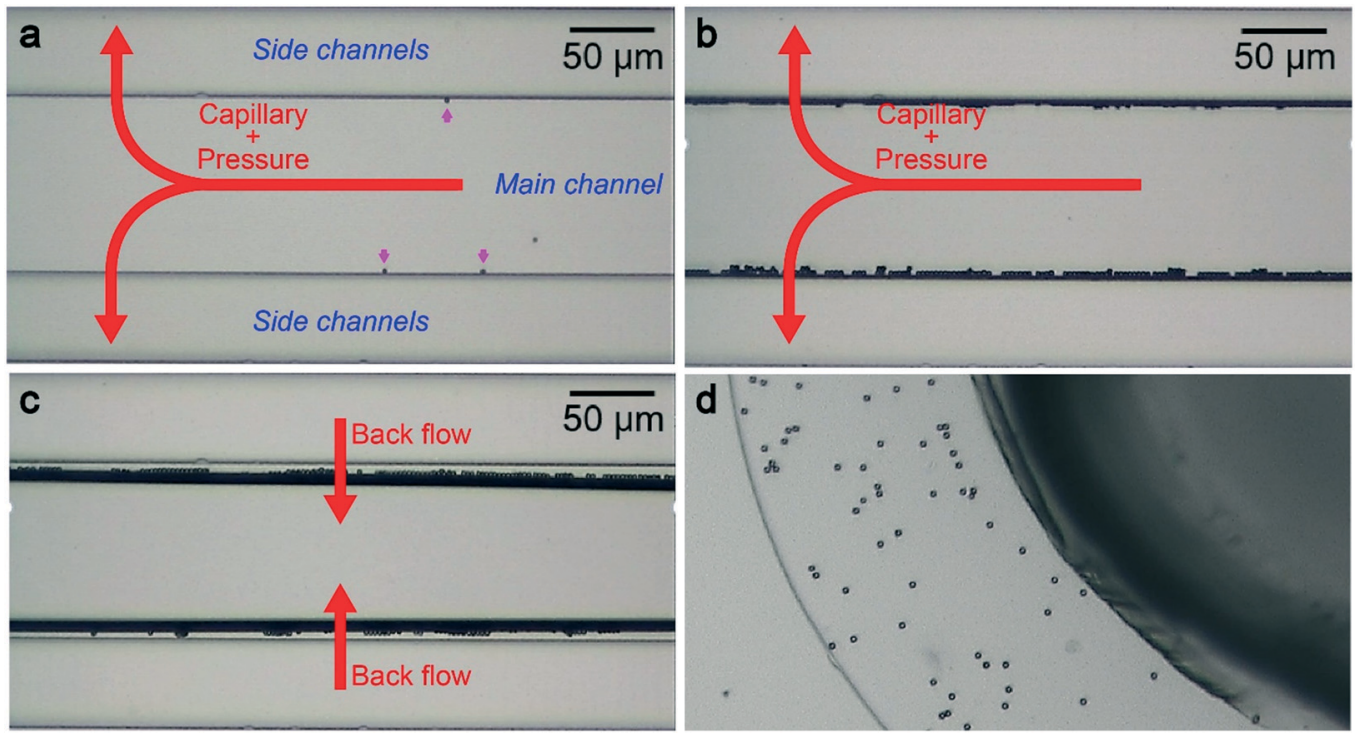

Fig. 3 Particle motions under fluid control. a, Optical image at an initial stage of the concentration process wherein the suspension of $6 \mu$ m-sized polystyrene beads was flowed through the side channels. Side channels are not visible. Arrows point to the polystyrene beads trapped at the orifices of the side-channels. b, After feeding the entire amount of solution, all the particles became trapped sporadically along both sides of the main channel. c, The trapped particles were then released by the back-flow through turning off the external pressure. Note that there are no particles left on the side surface indicating a negligible loss of analytes in the course of the concentration process. $d$, Particles transported to the pore chamber.

charges that contributed to induction of Coulomb repulsion against the negatively-charged polymeric microbeads. ${ }^{24,25}$ By this simple dry process, the particles eventually became completely removable leaving not a single bead unreleased at the side channels, which means $100 \%$ release efficiency. Finally, the released particles were sent to the micropore by flowing the buffer along the main channel (Fig. 3d, see also Movie S3 $\dagger$ ). These experiments prove the principle-ofoperation of the nanochannel-integrated pore for aggregation-free size-selective particle concentration with negligible loss.

\section{Rational design of side channels for rapid particle concentration}

Having demonstrated the particle concentration capability, it is important to explore an optimal channel design for rapid particle concentration. As mentioned already, the length of the side channels $L_{\mathrm{s}}$ is a crucial parameter determining the flow rate under a constant $P$. Hence we fabricated the side channels of $L_{\mathrm{s}}=30 \mu \mathrm{m}, 50 \mu \mathrm{m}$, and $100 \mu \mathrm{m}$ and recorded the time $t_{\text {drain }}$ required to drain $30 \mu \mathrm{L}$ of the particle solution. Here, we used particles of two different sizes, $6 \mu \mathrm{m}$ and $1 \mu \mathrm{m}$ in diameter, to verify the robustness of the method. As a result, we obtained a steady increase in $t_{\text {drain }}$ with $L_{\mathrm{s}}$ irrespective of the polymeric beads employed, which is in qualitative accordance with the Washburn model ${ }^{26}$ predicting the position $X$ of the moving meniscus at time $t$ as $X=(\gamma \cos$ $\theta h t / 3 \mu)^{0.5}$, where $\gamma$ is the surface tension of the liquid in air, $\theta$ is the contact angle of the liquid on the channel walls, $h$ is the channel height and $\mu$ is the fluid viscosity (Fig. 4a). Specifically, from this equation, we calculated the time $t_{\mathrm{ch}}$ re- quired for $X$ to be increased to $L_{\mathrm{s}}$, from which we deduced the flow speed $v_{\mathrm{ch}}$ as $L_{\mathrm{s}} / t_{\mathrm{ch}}$. Then, the flow rate was obtained as $N_{\mathrm{ch}} S v_{\mathrm{ch}}$ where $S$ is the cross-sectional area of the rectangular-shaped side channel and $N_{\mathrm{ch}}=660$ is the number of side channels. The results illustrate the effectiveness of utilizing short side channels to accelerate the concentration process.

\section{Deformation of the elastic channel}

Meanwhile, the drain time was found to be an order of magnitude longer than the theoretical expectation with $h=500$ $\mathrm{nm}$. On the other hand, the plots were close to the calculation when assuming much smaller channels of $h=150 \mathrm{~nm}$ (Fig. 4a). The lowered height most likely stems from the shrinkage of the conduit during the experiments due to mechanical deformation of ductile PDMS under the negative pressure. ${ }^{27}$ This could be resolved simply by replacing the rubber material with hard compounds such as glass ${ }^{28}$ and cyclo-olefin polymers. ${ }^{29}$ Nonetheless, these results serve as a guide to design appropriate side channels for completing the concentration process within $t_{\text {drain }}$ under a given $P$.

\section{Particle trapping mechanism}

The aggregation-free concentration is a key for post resistive pulse analysis,${ }^{11}$ which can be implemented only when particles are sporadically trapped along the main channel (Fig. 3b). For this, the main channel dimension has been arranged to the present size so as to provide an appropriate amount of space for the analytes to move through the conduit before being drawn into a side channel via the drag force. Meanwhile, $L_{\mathrm{s}}$ is also anticipated to affect the particle 
a

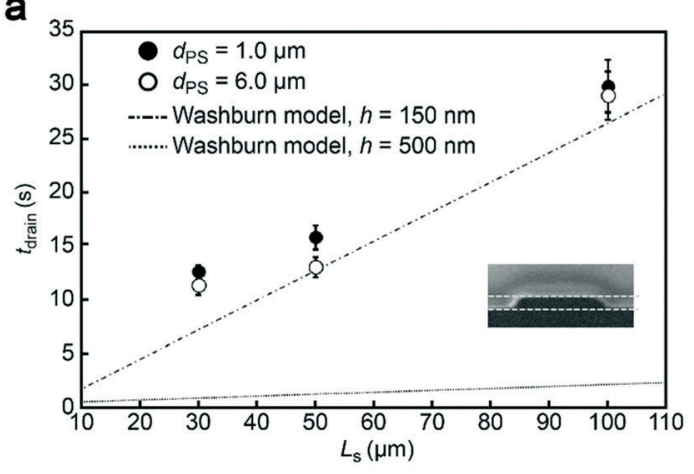

b

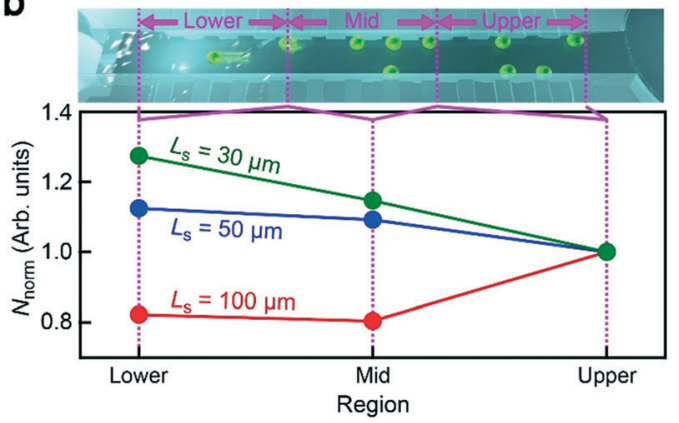

C

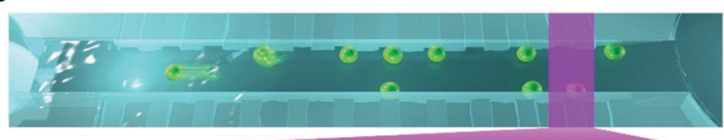

$L_{\mathrm{s}}=30 \mu \mathrm{m}$

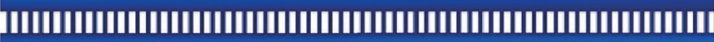

Main channel
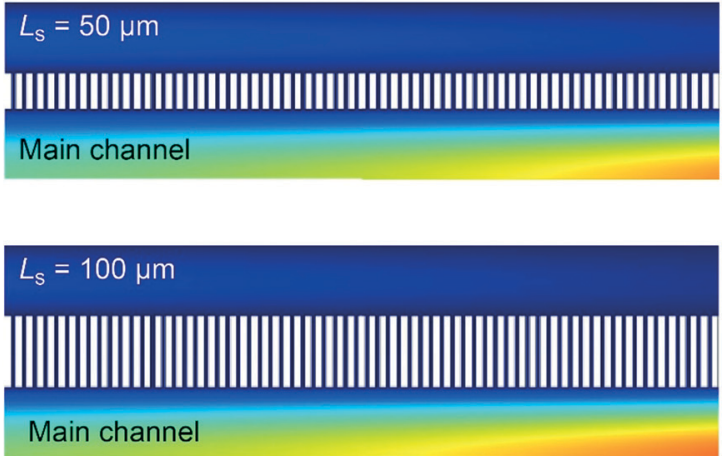

Fig. 4 Particle concentration mechanism. a, Time $t_{\text {drain }}$ required to flow $30 \mu \mathrm{L}$ of particle solution through the dual-height fluidic channels with side channel arrays of length $L_{s}$ under the negative pressure $-70 \mathrm{kPa}$. b. The position-dependent number of particles $N_{\text {norm }}$ normalized by that at the upstream part of the main channel (right side). c, Finite element analysis of the transverse fluid velocity along the side channels. Note that the velocity is higher at the upstream in the structure with longer side channels.

trapping dynamics through changing the transverse fluid flow rates: too long (short) $L_{\mathrm{s}}$ would be expected a priori to result in more frequent capturing of particles at the back (front) side of the main channel. To shed light on the concentration mechanism, therefore, we counted the number of trapped particles in three different regions from the front $(0$ $\mathrm{mm})$ to the back end $(6 \mathrm{~mm})$ of the main channel (Fig. $4 \mathrm{~b}$ ). Unexpectedly, by plotting the number of particles normalized by that at 0 to $2 \mathrm{~mm}$, it was revealed that more particles tended to be trapped at the back (front) side with shorter (longer) side channels.

To further study the counterintuitive $L_{\mathrm{s}}$ dependence of the trapped particle distributions, we performed numerical simulations of the fluid velocity $v_{\mathrm{w}}$ from the main to the side channels in a framework of Navier-Stokes theory with an assumption of lamellar flow. The $v_{\mathrm{w}}$ diagram illustrated faster convection at the main channel upstream in the case of longer $L_{\mathrm{s}}$ (Fig. $4 \mathrm{c}$ ). The associated hydrodynamic drag is, therefore, anticipated to bring a higher number of particles at the upstream of the $100 \mu \mathrm{m}$-long side channels than the other shorter ones, which is in qualitative accordance with the experimental observations (Fig. 4b).

\section{High-throughput single-bioparticle detections}

We evaluated the particle detection efficiency in the nanochannel-integrated micropores. We measured a dilute suspension of $1.0 \mu \mathrm{m}$-sized carboxylated polystyrene beads in $0.1 \times$ PBS at a concentration $c_{\mathrm{p}}$ of $10^{4}$ particles per $\mathrm{mL}$ with a micropore of $d_{\text {pore }}=1.5 \mu \mathrm{m}$. After the hydropressure-driven concentration by a nominal factor of 1000 (30 $\mu \mathrm{L}$ of the particle solution was flowed through the side channels followed by transportation to the pore chamber with $0.03 \mu \mathrm{L}$ buffer), we recorded the temporal change in the cross-pore ionic current $I_{\text {ion }}$ under a DC bias voltage of $0.1 \mathrm{~V}$. The $I_{\text {ion }}$ traces showed a pulse-like change in the current which is suggestive of electrophoretic translocation of the negatively-charged polymeric beads through the micropore (Fig. 5, trace in blue square). ${ }^{30}$ The frequency $f_{\mathrm{p}}$ used to observe an ionic spike per time, which is equivalent to the particle capture rate, was $0.31 \mathrm{~Hz}$ (Fig. 5, solid circle). This value was found to be considerably higher than that without the concentration showing the ionic spike-like signals at about 0.001 $\mathrm{Hz}$ at $10^{4}$ particles per $\mathrm{mL}$. Here, the enhancement ratio of 310 in $f_{\mathrm{p}}$ by the pre-concentration process represents a fair agreement with the nominal degree of particle concentration.

In order to verify the feasibility of the nanochannelintegrated-micropore sensor for bioanalytical applications, the resistive pulse measurements were extended to bacteria detections. We tested $E$. coli dispersed in $0.1 \times \mathrm{PBS}$ at $10^{3}$ cells per mL. After the 1000-fold concentration (see Movie S4 and S5 $\dagger$ showing, respectively, E. coli being trapped and released from a side channel), we acquired resistive pulses from which we deduced the bacterial capture rate of about $0.14 \mathrm{~Hz}$ (Fig. 5 


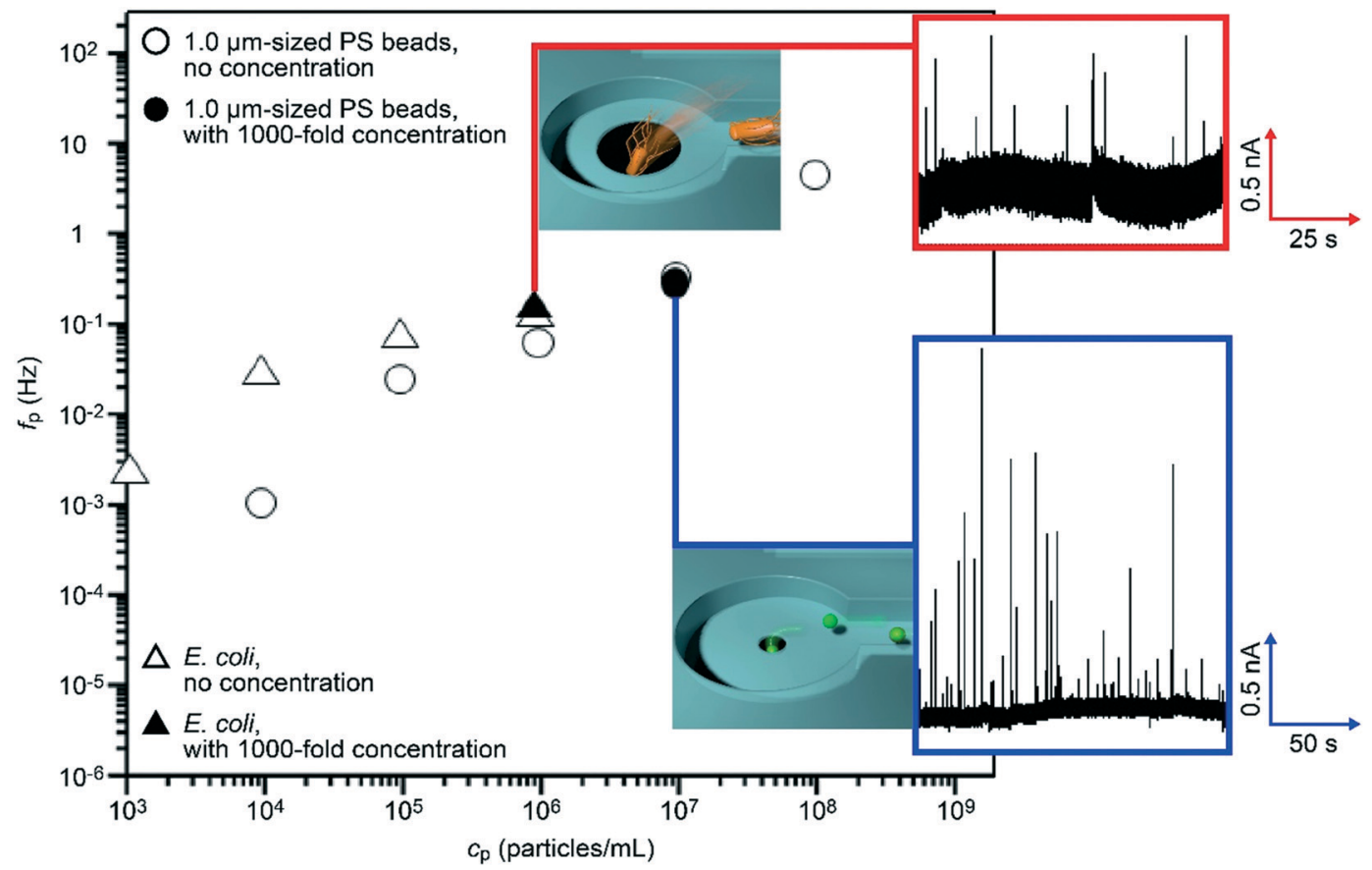

Fig. 5 High-throughput single-particle detections using dual-height-channel-integrated pore sensors. Detection frequency $f_{\mathrm{p}}$ plotted against the nominal particle concentration $c_{\mathrm{p}}$. Open and solid circles are the detection frequency of $1.0 \mu \mathrm{m}$-sized carboxylated polystyrene beads in $0.1 \times$ PBS using $1.5 \mu \mathrm{m}$ diameter pores without and with 1000-fold concentration via hydrodynamic control, respectively. Similar plots for $E$. coli in $0.1 \times$ PBS detected using $3.0 \mu \mathrm{m}$ diameter pores without and with 1000-fold concentration were shown by the open and solid triangles. The initial concentration conditions were, respectively, $10^{4}$ particles per $\mathrm{mL}$ (solid circle) and $10^{3}$ particles per $\mathrm{mL}$ (solid triangle) for the data of PS beads and $E$. coli.

, solid triangle). This value is to be compared to $f_{\mathrm{p}}=0.003 \mathrm{~Hz}$ at $c_{\mathrm{p}}=10^{3} \mathrm{~mL}^{-1}$ without the concentration suggesting an increase by a factor of 46 in the single-bacteria detection throughput. The fact that the number is lower than 1000 would be attributed to the presence of non-released cells being strongly adhered to the channel wall due to the rather hydrophobic nature of their surface. ${ }^{31}$ Nonetheless, the loss of detectable bacteria can be said as marginal. More importantly, we were able to continue the single-bacteria detections for more than 10 minutes without any clogging of the micropore owing to the aggregation-free concentration mechanism. The present results offer a proof-of-concept of highthroughput single-bioparticle detections using a nanochannel-integrated micropore that would find wide applications such as in environmental monitoring of water ${ }^{32}$ and bacterial screening of food. ${ }^{33,34}$

\section{Conclusions}

We developed a nanochannel-integrated micropore for high-throughput single-particle analyses via resistive pulse measurements. Using the device, we demonstrated aggregation-free particle concentration by a hydropressure-controlled trap-and-release mechanism at arrays of sub-particle-sized channels. This enabled three orders of magnitude enhancement in the throughput of resistive pulse detections of synthetic particles as well as bacterial cells.

\section{Conflicts of interest}

There are no conflicts to declare.

\section{Acknowledgements}

This work was supported by the ImPACT Program of Council for Science, Technology, and Innovation (Cabinet Office, Government of Japan).

\section{Notes and references}

1 S. Iqbal and R. Bashir, Nanopores: Sensing and Fundamental Biological Interactions, Springer, 2011.

2 W. Shi, A. K. Friedman and L. A. Baker, Anal. Chem., 2016, 89, 157-188.

3 L. Luo, S. R. German, W.-J. Lan, D. A. Holden, T. L. Mega and H. S. White, Annu. Rev. Anal. Chem., 2014, 7, 513-535.

4 D. Branton, D. W. Deamer, A. Marziali, H. Bayley, S. A. Benner, T. Butler, M. Di Ventra, S. Garaj, A. Hibbs, X. Huang, S. B. Jovanovich, P. S. Krstic, S. Lindsay, X. S. Ling, C. H. Mastrangelo, A. Meller, J. S. Oliver, Y. V. Pershin, J. M. Ramsey, R. Riehn, G. V. Soni, V. Tobard-Cossa, M. Wanunu, M. Wiggin and J. A. Schloss, Nat. Biotechnol., 2008, 26, 1146-1153.

5 S. Howorka and Z. Siwy, Chem. Soc. Rev., 2009, 38, 2360-2384.

6 D. G. Haywood, A. Saha-Shah, L. A. Baker and S. C. Jacobson, Anal. Chem., 2011, 6, 615-624. 
7 W. H. Coulter, US Pat., 2656508, 1953.

8 R. Vogel, G. Willmott, D. Kozak, S. Roberts, W. Anderson, L. Groenewegen, B. Glossop, A. Barnett, A. Turner and M. Trau, Anal. Chem., 2011, 83, 3499-3506.

9 H. Yasaki, T. Yasui, T. Yanagida, N. Kaji, M. Kanai, K. Nagashima, T. Kawai and Y. Baba, J. Am. Chem. Soc., 2017, 139, 14137-14142.

10 E. E. Sheehan and L. J. Whitman, Nano Lett., 2005, 5, 803-807.

11 K. J. Freedman, M. Jurgens, A. Prabhu, C. W. Ahn, P. Jemth, J. B. Edel and M. J. Kim, Anal. Chem., 2011, 83, 5137-5144.

12 M. A. Edwards, S. R. German, J. E. Dick, A. J. Bard and H. White, ACS Nano, 2015, 9, 12274-12282.

13 R. Torre, J. Larkin, A. Singer and A. Meller, Nanotechnology, 2012, 23, 385308.

14 M. Tsutsui, K. Yokota, T. Nakada, A. Arima, W. Tonomura, M. Taniguchi, T. Washio and T. Kawai, ACS Sens., 2018, 3, 2693-2701.

15 Y. He, M. Tsutsui, C. Fan, M. Taniguchi and T. Kawai, ACS Nano, 2011, 5, 8391-8397.

16 K. J. Freedman, L. M. Otto, A. P. Ivanov, A. Barik, S.-H. Oh and J. B. Edel, Nat. Commun., 2016, 7, 10217.

17 N. D. Fiori, A. Squires, D. Bar, T. Gilboa, T. D. Moustakas and A. Meller, Nat. Nanotechnol., 2013, 8, 946-951.

18 Y. He, M. Tsutsui, R. H. Scheicher, F. Bai, M. Taniguchi and T. Kawai, ACS Nano, 2013, 7, 538-546.

19 M. Zhang, C. Ngampeerapong, D. Radin, A. Ahmadian, I. Sychugov and J. Linnros, ACS Sens., 2018, 12, 4574-4582.
20 M. Wanunu, W. Morrison, R. Rabin, A. Y. Grosberg and A. Meller, Nat. Nanotechnol., 2010, 5, 160-165.

21 J.-L. Fraikin, T. Teesalu, C. M. McKenney, E. Ruoslahti and A. N. Cleland, Nat. Nanotechnol., 2011, 6, 308-313.

22 M. N. Hamblin, J. Xuan, D. Maynes, H. D. Tolley, D. M. Belnap, A. T. Woolley, M. L. Lee and A. R. Hawkins, Lab Chip, 2010, 10, 173-178.

23 D. T. Eddington, J. P. Puccinelli and D. J. Beebe, Sens. Actuators, B, 2006, 114, 170-172.

24 M. Mrra, E. Occhiello, R. Marola, F. Garbassi, P. Humphrey and D. Johnson, J. Colloid Interface Sci., 1990, 137, 11-24.

25 S. Bhattacharaya, A. Datta, J. M. Berg and S. Gangopadhyay, J. Microelectromech. Syst., 2005, 14, 590-597.

26 E. W. Washburn, Phys. Rev., 1921, 17, 273-278.

27 B.-Y. Xu, X.-N. Yan, J.-D. Zhang, J.-J. Xu and H.-Y. Chen, Lab Chip, 2012, 12, 381-386.

28 P. Mao and J. Han, Lab Chip, 2005, 5, 837-844.

29 L. Yi, W. Xiaodong and Y. Fan, J. Mater. Process. Technol., 2008, 208, 63-69.

30 M. Tsutsui, Y. He, K. Yokota, A. Arima, S. Hongo, M. Taniguchi, T. Washio and T. Kawai, ACS Nano, 2016, 10, 803-809.

31 M. Rosenberg, D. Gutnick and E. Rosenberg, FEMS Microbiol. Lett., 1980, 9, 29-33.

32 J. E. Hobbie, R. J. Daley and S. Jasper, Appl. Environ. Microbiol., 1977, 33, 1225-1228.

33 S. Tokonami, E. Shimizu, M. Tamura and T. Iida, Sci. Rep., 2017, 7, 16651.

34 I. Percin, N. Idil, M. Bakhshpour, E. Yilmaz, B. Mattiasson and A. Denizli, Sensors, 2017, 17, 1375. 